Dokuz Eylül Üniversitesi-Mühendislik Fakültesi Fen ve Mühendislik Dergisi

Cilt 19, Sayı 57, Eylül 2017
Dokuz Eylul University-Faculty of Engineering Journal of Science and Engineering Volume 19, Issue 57, September 2017

DOI: $10.21205 /$ deufmd.2017195774

\title{
Schur Complements of Block Kronecker Products
}

\section{Mustafa ÖZEL ${ }^{* 1}$}

${ }^{1}$ Dokuz Eylül Üniversitesi, Mühendislik Fakültesi, Jeofizik Bölümü, 35160, İzmir

(Alınış / Received: 23.12.2017, Kabul / Accepted: 18.05.2017, Online Yayınlanma / Published Online: 20.09.2017)

\section{Keywords}

Schur

Complements, Block Kronecker Product, Positive Semidefinite Matrices, MoorePenrose Inverse
Abstract: The paper is established on Schur complements and block Kronecker product of positive semidefinite matrices. In particular, a formulation for the block Kronecker product of Schur complements of block matrices is improved. Additionally, an new following inequality for block Kronecker product of Schur complements of two matrices and their conjugate transpose is proved

$$
[(A / \alpha) \otimes(B / \beta)][(A / \alpha) \otimes(B / \beta)]^{*} \geq\left[(A \otimes B)(A \otimes B)^{*}\right] / \gamma .
$$

\section{Blok Kronecker Çarpımların Schur Tamamlayıcıları}

\section{Anahtar \\ Kelimeler \\ Schur \\ Tamamlayıcılar, Blok Kronecker \\ Çarpım, Yarı \\ Pozitif Tanımlı \\ Matrices , \\ Moore-Penrose \\ Tersler}

Özet: $\mathrm{Bu}$ çalışma, yarı pozitif tanımlı blok matrislerin blok kronecker çarpımları ve Schur tamamlayıcıları üzerine kurulmuştur. Özellikle blok matrislerin Schur tamamlayıcılarının blok kronecker çarpımı için bir formülasyon geliştirilmiştir. Bundan başka, iki matris ve eşlenik transpozlarının schur tamamlayıcılarının blok kronecker çarpımları için aşağıdaki yeni eşitsizlik kanıtlanmıştır.

$$
[(\boldsymbol{A} / \boldsymbol{\alpha}) \otimes(B / \beta)][(\boldsymbol{A} / \boldsymbol{\alpha}) \otimes(B / \beta)]^{*} \geq\left[(\boldsymbol{A} \otimes \boldsymbol{B})(\boldsymbol{A} \otimes B)^{*}\right] / \gamma
$$




\section{Introduction}

The Kronecker product has many practical applications in system theory including the analysis of stochastic steady state, matrix equations, matrix calculus, signal and image processing, and statistical mechanics[1]. Furthermore the spectral theorem for finite dimensional Hermitian matrices can be formulated using the Kronecker product.

In this paper, we derive an inequality relating the Schur complements of the block Kronecker product for the block matrices. The inequality had been known for usual Kronecker product, so the main contribution here is the extension to the block Kronecker product which was defined by Horn, Mathias, and Nakamura[2]. Then, some of its properties were improved by Günther and Klotz [3].

Let $M_{m, n}$ denote the space of $m \times n$ complex matrices and $\boldsymbol{M}_{p, q}\left(M_{m, n}\right)$ denote the space of $p \times q$ block matrices $\boldsymbol{A}=\left(A_{i j}\right)_{i=1, \ldots, p}^{j=1, \ldots, q}$ whose $i, j$ entry belongs to $M_{m, n}$ for the positive integers $p, q, m$, and $n$. We write $M_{n} \equiv M_{n, n}$ and $\boldsymbol{M}_{\boldsymbol{p}} \equiv \boldsymbol{M}_{p, p}$. Also The identity matrix in is $M_{p}\left(M_{n}\right)$ is denoted $I_{n}$. If $\boldsymbol{A}>0(\geq 0)$, we say $\boldsymbol{A}$ is positive(positive semi) definite and if $\boldsymbol{A}>B(\geq 0)$, we say $\boldsymbol{A}-\boldsymbol{B}>$ $0(\geq 0)$ for positive(positive semi) definite matrices $\boldsymbol{A}$ and $\boldsymbol{B}$.

Let $\boldsymbol{\alpha} \subseteq\{1,2, \ldots, p\}, \boldsymbol{\beta} \subseteq\{1,2, \ldots, q\}$ be the index sets and $\boldsymbol{\alpha}^{c}=\{1,2, \ldots, p\} \backslash \boldsymbol{\alpha}, \boldsymbol{\beta}^{c}=$ $\{1,2, \ldots, q\} \backslash \boldsymbol{\beta}$ be the complements of $\boldsymbol{\alpha}$ and $\boldsymbol{\beta}$, and their cardinalities are $|\boldsymbol{\alpha}|$ and $|\boldsymbol{\beta}|$. Also we denote by $\boldsymbol{A}(\boldsymbol{\alpha}, \boldsymbol{\beta})$ that block submatrix of $\boldsymbol{A}$ with the block rows indexed by $\boldsymbol{\alpha}$ and the block columns indexed by $\boldsymbol{\beta}$. We usually write $\boldsymbol{A}(\boldsymbol{\alpha})$ for $\boldsymbol{A}(\boldsymbol{\alpha}, \boldsymbol{\alpha})$.
For a matrix we denote by $\boldsymbol{A}^{*}, \boldsymbol{A}^{-1}$ and $\boldsymbol{A}^{+}$ its conjugate transpose, inverse and Moore-Penrose inverse, respectively.

\section{Preliminaries}

We introduce in this section the main definition of the paper, that is, the block Kronecker product of two matrices and also give some properties of its.

Definition 2.1. Let $A \in M_{m, l}$ and $\boldsymbol{B}=$ $\left(B_{i j}\right) \in M_{s, t}\left(M_{l, n}\right)$. Then the block Kronecker product $A$ and $\boldsymbol{B}$ is defined by

$$
A \bigotimes \boldsymbol{B}=\left(A B_{i j}\right)_{i=1, \ldots, S}^{j=1, \ldots, t}
$$

where $A B_{i j}$ is the usual matrix product of $A$ and $B_{i j}$. For $\boldsymbol{A}=\left(A_{i j}\right) \in M_{p, q}\left(M_{m, l}\right)$, the block Kronecker product is given by

$$
\boldsymbol{A} \bigotimes \boldsymbol{B}=\left(A_{i j} \otimes \boldsymbol{B}\right)_{i=1, \ldots, p}^{j=1, \ldots, q}
$$

Definition 2.2. If every $n \times n$ block of $\boldsymbol{A}$ commutes with every $n \times n$ block of $\boldsymbol{B}$ then the block matrices $\boldsymbol{A} \in M_{p, q}$ and $\boldsymbol{B} \in M_{s, t}$ are called as block commuting and denoted by $\boldsymbol{A}_{b c} \boldsymbol{B}$. [3]

Definition 2.3. If $|\boldsymbol{\alpha}|=|\boldsymbol{\beta}|$ and $\boldsymbol{A}(\boldsymbol{\alpha}, \boldsymbol{\beta})$ is nonsingular then the block of the Schur complement of $\boldsymbol{A}(\boldsymbol{\alpha}, \boldsymbol{\beta})$ in $\boldsymbol{A}$ is

$$
\begin{aligned}
& \boldsymbol{A} / \boldsymbol{A}(\boldsymbol{\alpha}, \boldsymbol{\beta}) \\
& =\boldsymbol{A}\left(\boldsymbol{\alpha}^{c}, \boldsymbol{\beta}^{c}\right)-\boldsymbol{A}\left(\boldsymbol{\alpha}^{c}, \boldsymbol{\beta}\right)(\boldsymbol{A}(\boldsymbol{\alpha}, \boldsymbol{\beta}))^{-1} \boldsymbol{A}\left(\boldsymbol{\alpha}, \boldsymbol{\beta}^{c}\right)
\end{aligned}
$$

It is useful to denote $\boldsymbol{A} / \boldsymbol{\alpha}$ for $\boldsymbol{A} / \boldsymbol{A}(\boldsymbol{\alpha})$. [46]

Lemma 2.4. (a) $(\boldsymbol{A} \bigotimes \boldsymbol{B})^{*}=\boldsymbol{A}^{*} \bigotimes \boldsymbol{B}^{*}$ if and only if $\boldsymbol{A}_{b c} \boldsymbol{B}$.

(b) If $\boldsymbol{B}_{b c} \boldsymbol{C}$ then

$(A \otimes B)(C \otimes D)=A C \bigotimes B D$.

(c) If $\boldsymbol{A}_{b c} \boldsymbol{B}^{*}$ then $(\boldsymbol{A} \otimes \boldsymbol{B})^{+}=\boldsymbol{A}^{+} \otimes \boldsymbol{B}^{+}$. 
(d) If $\boldsymbol{A}_{b c} \boldsymbol{B}$ and $\boldsymbol{A}_{b c} \boldsymbol{B}^{*}$, then $\boldsymbol{A}_{b c} \boldsymbol{B}^{+}$. [3]

\section{On Inequalities for Block Kronecker Products}

In this section, we present some inequalities involving the block Kronecker product of positive definite block matrices and its Schur complements.

Lemma 3.1. Let $A \geq C \geq 0, \quad B \geq D \geq 0$, $\boldsymbol{A}_{b c} \boldsymbol{B}$ and $\boldsymbol{A}_{b c} \boldsymbol{B}^{*}$, and $\boldsymbol{A}, \boldsymbol{B}, \boldsymbol{C}$, and $\boldsymbol{D}$ be compatibly block matrices, then

$$
\boldsymbol{A} \otimes \boldsymbol{B} \geq \boldsymbol{C} \otimes \boldsymbol{D}
$$

Proof. To constitute $\mathrm{Eq}(1)$ we first take $\boldsymbol{A} \otimes \boldsymbol{B}$ with $A_{11} \otimes \boldsymbol{B}$;

$$
\begin{aligned}
& \boldsymbol{A} \otimes \boldsymbol{B}=\left[\begin{array}{ll}
A_{11} \otimes \boldsymbol{B} & A_{12} \otimes \boldsymbol{B} \\
A_{12}^{*} \otimes \boldsymbol{B} & A_{22} \otimes \boldsymbol{B}
\end{array}\right], \\
& A_{11} \otimes \boldsymbol{B}=\left[\begin{array}{ll}
A_{11} B_{11} & A_{11} B_{12} \\
A_{11} B_{12}^{*} & A_{11} B_{22}
\end{array}\right] .
\end{aligned}
$$

We will prove the following three statements by taking into consideration the above factorization.

(i) $A_{11} \otimes \boldsymbol{B} \geq \mathbf{0}$. Since $\boldsymbol{B} \geq \mathbf{0}$, by using the Albert's theorem [7] we write

and

$$
B_{11} \geq 0, B_{22}-B_{12}^{*} B_{22}^{+} B_{12} \geq 0
$$

$$
B_{12}=B_{11} B_{11}^{+} B_{12} .
$$

From the hypothesis $\boldsymbol{A}$ and $\boldsymbol{B}$ are positive semidefinite and block commute, $A_{11} B_{11} \geq 0$. By using Lemma 2.4

$$
\begin{aligned}
A_{11} B_{22} & -A_{11} B_{12}^{*}\left(A_{11} B_{11}\right)^{+}\left(A_{11} B_{12}\right) \\
& =A_{11} B_{22}-A_{11} B_{12}^{*} B_{11}^{+} A_{11}^{+} A_{11} B_{12} \\
& =A_{11} B_{22}-A_{11} A_{11}^{+} A_{11} B_{12}^{*} B_{11}^{+} B_{12} \\
& =A_{11}\left(B_{22}-B_{12}^{*} B_{11}^{+} B_{12}\right) \geq 0
\end{aligned}
$$

is obtained. Then, by second condition of Albert's theorem and Lemma 2.4,

$$
\begin{gathered}
A_{11} B_{12}^{*}=\left(A_{11} B_{11}\right)\left(A_{11} B_{11}\right)^{+}\left(A_{11} B_{12}\right) \\
=A_{11} B_{11} B_{11}^{+} A_{11}^{+} A_{11} B_{12} \\
=A_{11} A_{11}^{+} A_{11} B_{11} B_{11}^{+} B_{12} \\
=A_{11} B_{11} B_{11}^{+} B_{12} .
\end{gathered}
$$

(ii) Now we consider the second statement for $\boldsymbol{A} \otimes \boldsymbol{B}$

$A_{22} \otimes \boldsymbol{B}-\left(A_{12}^{*} \otimes \boldsymbol{B}\right)\left(A_{11} \otimes \boldsymbol{B}\right)^{+}\left(A_{12} \otimes \boldsymbol{B}\right) \geq 0$.

To prove this inequality we also use Lemma 2.4

$$
\begin{aligned}
& A_{22} \otimes \boldsymbol{B}-\left(A_{12}^{*} \otimes \boldsymbol{B}\right)\left(A_{11} \otimes \boldsymbol{B}\right)^{+}\left(A_{12} \otimes \boldsymbol{B}\right) \\
& =A_{22} \otimes \boldsymbol{B}-A_{12}^{*} A_{11}^{+} A_{12} \otimes \boldsymbol{B} \boldsymbol{B}^{+} \boldsymbol{B} \\
& =\left(A_{22}-A_{12}^{*} A_{11}^{+} A_{12}\right) \otimes \boldsymbol{B}
\end{aligned}
$$

and the matrix $\boldsymbol{A}$ ensures the Albert's theorem [7], so the second statement is proved.

(iii) For

$A_{12} \otimes \boldsymbol{B}=\left(A_{11} \otimes \boldsymbol{B}\right)\left(A_{11} \otimes \boldsymbol{B}\right)^{+}\left(A_{12} \otimes \boldsymbol{B}\right)$, by Lemma 2.4

$A_{12} \otimes \boldsymbol{B}=\left(A_{11} \otimes \boldsymbol{B}\right)\left(A_{11}^{+} \otimes \boldsymbol{B}^{+}\right)\left(A_{12} \otimes \boldsymbol{B}\right)$

$$
\begin{aligned}
& =A_{11} A_{11}^{+} A_{12} \otimes \boldsymbol{B} \boldsymbol{B}^{+} \boldsymbol{B} \\
& =A_{11} A_{11}^{+} A_{12} \otimes \boldsymbol{B} .
\end{aligned}
$$

By taking into consideration Albert's theorem[7] based on (i), (ii), and (iii) we get

$\boldsymbol{A} \otimes \boldsymbol{B} \geq \mathbf{0}$ for $\boldsymbol{A} \geq 0$ and $\boldsymbol{B} \geq 0$

Furthermore, this results implies that $C \otimes D \geq 0$, 


$$
\begin{aligned}
& A \otimes B-C \otimes D=A \otimes B-A \otimes D+A \otimes D \\
& \begin{array}{c}
-C \otimes D \\
=A(B-D)+(A-C) \otimes D \geq 0
\end{array}
\end{aligned}
$$

Hence, $\boldsymbol{A} \geq \boldsymbol{C}$ and $\boldsymbol{B} \geq \boldsymbol{D}$.

Theorem 3.2. Let $\boldsymbol{A} \in M_{p}\left(M_{n}\right)$, and $\boldsymbol{B} \in M_{q}\left(M_{n}\right)$ and $\boldsymbol{A}_{b c} \boldsymbol{B}, \boldsymbol{\alpha} \subseteq\{1,2, \ldots, p\}$, $\boldsymbol{\beta} \subseteq\{1,2, \ldots, q\}, \boldsymbol{\alpha}^{c}=\{1,2, \ldots, p\} \backslash \boldsymbol{\alpha}, \boldsymbol{\beta}^{c}=$ $\{1,2, \ldots, q\} \backslash \boldsymbol{\beta}$ and $\boldsymbol{\gamma}=\{1,2, \ldots, p\} \backslash \boldsymbol{\gamma}^{c}$ where $\boldsymbol{\gamma}^{c}=\left\{q(i-1)+j, \quad i \in \boldsymbol{\alpha}^{c}, j \in\right.$ $\left.\boldsymbol{\beta}^{c}\right\}$. Then

$$
(A / \alpha) \otimes(B / \beta)=(A \otimes B) / \gamma
$$

Proof. If $A(\alpha)$ is nonsingular then $\boldsymbol{A} / \boldsymbol{\alpha}$ is nonsingular since $A$ is nonsingular by Schur's determinant formula. Therefore

Hence,

$$
(\boldsymbol{A} / \alpha)^{-1}=\boldsymbol{A}^{-1}\left(\boldsymbol{\alpha}^{c}\right)
$$

$$
\begin{aligned}
(A / \alpha)^{-1} \otimes(\boldsymbol{B} / \boldsymbol{\beta})^{-1} & =\left[\boldsymbol{A}^{-1}\left(\boldsymbol{\alpha}^{c}\right) \otimes \boldsymbol{B}^{-1}\left(\boldsymbol{\beta}^{c}\right)\right] \\
& =\left(\boldsymbol{A}^{-1} \otimes \boldsymbol{B}^{-1}\right)\left(\gamma^{c}\right)
\end{aligned}
$$

by using Lemma2.4 (c) and Eq(3)

$$
\begin{aligned}
(\boldsymbol{A} / \boldsymbol{\alpha}) \otimes(B) & =\left[(\boldsymbol{A} / \boldsymbol{\alpha})^{-1} \otimes(\boldsymbol{B} / \boldsymbol{\beta})^{-1}\right]^{-1} \\
& =\left[\left(\boldsymbol{A}^{-1} \otimes \boldsymbol{B}^{-1}\right)\left(\boldsymbol{\gamma}^{c}\right)\right]^{-1} \\
& =\left[(\boldsymbol{A} \otimes \boldsymbol{B})^{-1}\left(\gamma^{c}\right)\right]^{-1} \\
& =\left\{[(\boldsymbol{A} \otimes \boldsymbol{B}) / \gamma]^{-1}\right\}^{-1} \\
& =(\boldsymbol{A} \otimes \boldsymbol{B}) / \gamma \cdot \square
\end{aligned}
$$

Theorem 3.3. Let $\boldsymbol{A} \in M_{p}\left(M_{n}\right)$, and $\boldsymbol{B} \in M_{q}\left(M_{n}\right)$ and $\boldsymbol{A}_{b c} \boldsymbol{B}, \boldsymbol{\alpha} \subseteq\{1,2, \ldots, p\}$, $\boldsymbol{\beta} \subseteq\{1,2, \ldots, q\}, \boldsymbol{\alpha}^{c}=\{1,2, \ldots, p\} \backslash \boldsymbol{\alpha}, \boldsymbol{\beta}^{c}=$ $\{1,2, \ldots, q\} \backslash \boldsymbol{\beta}$ and $\boldsymbol{\gamma}=\{1,2, \ldots, p\} \backslash \gamma^{c}$

where

$$
\boldsymbol{\gamma}^{c}=\left\{q(i-1)+j, \quad i \in \boldsymbol{\alpha}^{c}, \quad j \in \boldsymbol{\beta}^{c}\right\} .
$$

Then

$$
\begin{aligned}
{[(\boldsymbol{A} / \boldsymbol{\alpha}) \otimes(B / \beta)] } & {[(\boldsymbol{A} / \boldsymbol{\alpha}) \otimes(B / \beta)]^{*} } \\
\geq & {\left[(\boldsymbol{A} \otimes B)(\boldsymbol{A} \otimes B)^{*}\right] / \gamma }
\end{aligned}
$$

Proof. For the convenient to think of the Schur complement of $\boldsymbol{A}$ as being in the upper left corner of $\boldsymbol{A}$ we use the permuation matrices $\boldsymbol{P}$ and $\boldsymbol{Q}$ for this placement, such that

$$
P A Q^{*}=\left[\begin{array}{cc}
A(\alpha) & A\left(\alpha, \alpha^{c}\right) \\
A\left(\alpha^{c}, \alpha\right) & A\left(\alpha^{c}, \alpha^{c}\right)
\end{array}\right]
$$

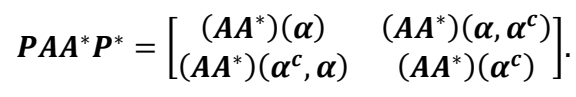

Let $\bar{\alpha}=\{1,2, \cdots,|\alpha|\}$ then

$\boldsymbol{P} \boldsymbol{A} \boldsymbol{Q}^{*} /_{\bar{\alpha}}=A / \boldsymbol{\alpha}$ and $\boldsymbol{P A A ^ { * } \boldsymbol { P } ^ { * }} /_{\bar{\alpha}}=\left(\boldsymbol{A A ^ { * } )} / \boldsymbol{\alpha}\right.$.

Thus, assuming that $\alpha=\{1,2, \cdots,|\alpha|\}$ and choosing

$$
\begin{aligned}
\boldsymbol{X}=-\boldsymbol{A}\left(\boldsymbol{\alpha}^{c}, \boldsymbol{\alpha}\right)[\boldsymbol{A}(\boldsymbol{\alpha})]^{-1} \text { and } \\
\quad \boldsymbol{Y}=-\left[\left(\boldsymbol{A} \boldsymbol{A}^{*}\right)\left(\boldsymbol{\alpha}^{c}, \boldsymbol{\alpha}\right)\right]\left[\left(\boldsymbol{A} \boldsymbol{A}^{*}\right)(\boldsymbol{\alpha})\right]^{-1}
\end{aligned}
$$

then

$$
(X, I) A=(0, A / \alpha)
$$

We have

$$
\begin{gathered}
(\boldsymbol{A} / \boldsymbol{\alpha})(\boldsymbol{A} / \boldsymbol{\alpha})^{*}=\left(0, \begin{array}{c}
\boldsymbol{A} / \boldsymbol{\alpha})(0, \boldsymbol{A} / \boldsymbol{\alpha})^{*} \\
=(\boldsymbol{X}, \boldsymbol{I})\left(\boldsymbol{A} \boldsymbol{A}^{*}\right)(\boldsymbol{X}, \boldsymbol{I})^{*}
\end{array}\right. \\
=(\boldsymbol{X}, \boldsymbol{I})\left(\begin{array}{cc}
\left(\boldsymbol{A} \boldsymbol{A}^{*}\right)(\boldsymbol{\alpha}) & \left(\boldsymbol{A} \boldsymbol{A}^{*}\right)\left(\boldsymbol{\alpha}, \boldsymbol{\alpha}^{c}\right) \\
\left(\boldsymbol{A} \boldsymbol{A}^{*}\right)\left(\boldsymbol{\alpha}^{\boldsymbol{c}}, \boldsymbol{\alpha}\right) & \left(\boldsymbol{A} \boldsymbol{A}^{*}\right)\left(\boldsymbol{\alpha}^{c}\right)
\end{array}\right)\left(\begin{array}{c}
\boldsymbol{X}^{*} \\
\boldsymbol{I}
\end{array}\right)
\end{gathered}
$$




$$
\begin{aligned}
& =\left(\boldsymbol{X}\left[\left(\boldsymbol{A} \boldsymbol{A}^{*}\right)(\boldsymbol{\alpha})\right]+\left(\boldsymbol{A} \boldsymbol{A}^{*}\right)\left(\boldsymbol{\alpha}^{c}, \boldsymbol{\alpha}\right), \boldsymbol{X}\left[\left(\boldsymbol{A} \boldsymbol{A}^{*}\right)\left(\boldsymbol{\alpha}, \boldsymbol{\alpha}^{c}\right)\right]+\left(\boldsymbol{A} \boldsymbol{A}^{*}\right)\left(\boldsymbol{\alpha}^{c}\right)\right)\left(\begin{array}{c}
\boldsymbol{X}^{*} \\
\boldsymbol{I}
\end{array}\right) \\
& =\left(\boldsymbol{A} \boldsymbol{A}^{*}\right)\left(\boldsymbol{\alpha}^{c}\right)+\boldsymbol{X}\left[\left(\boldsymbol{A} \boldsymbol{A}^{*}\right)\left(\boldsymbol{\alpha}, \boldsymbol{\alpha}^{c}\right)\right]+\left[\left(\boldsymbol{A} \boldsymbol{A}^{*}\right)\left(\boldsymbol{\alpha}^{c}, \boldsymbol{\alpha}\right)\right] \boldsymbol{X}^{*}+\boldsymbol{X}\left[\left(\boldsymbol{A} \boldsymbol{A}^{*}\right)(\boldsymbol{\alpha})\right] \boldsymbol{X}^{*}
\end{aligned}
$$

Since

$$
\left(\boldsymbol{A} \boldsymbol{A}^{*}\right) / \boldsymbol{\alpha}=\left(\boldsymbol{A} \boldsymbol{A}^{*}\right)\left(\boldsymbol{\alpha}^{c}\right)-\left(\boldsymbol{A A ^ { * }}\right)\left(\boldsymbol{\alpha}^{c}, \boldsymbol{\alpha}\right)\left[\left(\boldsymbol{A A ^ { * }}\right)(\boldsymbol{\alpha})\right]^{-1}\left[\left(\boldsymbol{A} \boldsymbol{A}^{*}\right)\left(\boldsymbol{\alpha}, \boldsymbol{\alpha}^{c}\right)\right]
$$

we have

$$
\begin{aligned}
(\boldsymbol{A} / \boldsymbol{\alpha})(\boldsymbol{A} / \boldsymbol{\alpha})^{*}= & \left(\boldsymbol{A} \boldsymbol{A}^{*}\right) / \alpha+\left(\boldsymbol{A} \boldsymbol{A}^{*}\right)\left(\boldsymbol{\alpha}^{c}, \boldsymbol{\alpha}\right)\left[\left(\boldsymbol{A} \boldsymbol{A}^{*}\right)(\boldsymbol{\alpha})\right]^{-1}\left[\left(\boldsymbol{A} \boldsymbol{A}^{*}\right)\left(\boldsymbol{\alpha}, \boldsymbol{\alpha}^{c}\right)\right] \\
& +\boldsymbol{X}\left[\left(\boldsymbol{A} \boldsymbol{A}^{*}\right)\left(\boldsymbol{\alpha}, \boldsymbol{\alpha}^{c}\right)\right]+\left[\left(\boldsymbol{A} \boldsymbol{A}^{*}\right)\left(\boldsymbol{\alpha}^{c}, \boldsymbol{\alpha}\right)\right] \boldsymbol{X}^{*}+\boldsymbol{X}\left[\left(\boldsymbol{A} \boldsymbol{A}^{*}\right)(\boldsymbol{\alpha})\right] \boldsymbol{X}^{*} \\
= & \left(\boldsymbol{A} \boldsymbol{A}^{*}\right) / \alpha+(\boldsymbol{X}-\boldsymbol{Y})\left[\left(\boldsymbol{A} \boldsymbol{A}^{*}\right)\left(\boldsymbol{\alpha}, \boldsymbol{\alpha}^{c}\right)\right]+\left[\left(\boldsymbol{A} \boldsymbol{A}^{*}\right)\left(\boldsymbol{\alpha}^{c}, \boldsymbol{\alpha}\right)\right] \boldsymbol{X}^{*}+\boldsymbol{X}\left[\left(\boldsymbol{A} \boldsymbol{A}^{*}\right)(\boldsymbol{\alpha})\right] \boldsymbol{X}^{*} \\
= & \left(\boldsymbol{A} \boldsymbol{A}^{*}\right) / \alpha+(\boldsymbol{X}-\boldsymbol{Y})\left[\left(\boldsymbol{A} \boldsymbol{A}^{*}\right)(\boldsymbol{\alpha})\right]\left[\left(\boldsymbol{A} \boldsymbol{A}^{*}\right)(\boldsymbol{\alpha})\right]^{-1}\left[\left(\boldsymbol{A} \boldsymbol{A}^{*}\right)\left(\boldsymbol{\alpha}, \boldsymbol{\alpha}^{c}\right)\right] \\
& +\left[\left(\boldsymbol{A} \boldsymbol{A}^{*}\right)\left(\boldsymbol{\alpha}^{c}, \boldsymbol{\alpha}\right)\right]\left[\left(\boldsymbol{A} \boldsymbol{A}^{*}\right)(\boldsymbol{\alpha})\right]^{-1}\left[\left(\boldsymbol{A} \boldsymbol{A}^{*}\right)(\boldsymbol{\alpha})\right] \boldsymbol{X}^{*}+\boldsymbol{X}\left[\left(\boldsymbol{A} \boldsymbol{A}^{*}\right)(\boldsymbol{\alpha})\right] \boldsymbol{X}^{*}
\end{aligned}
$$

using Eq(5)

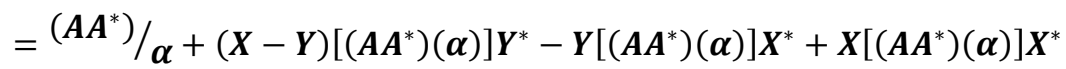

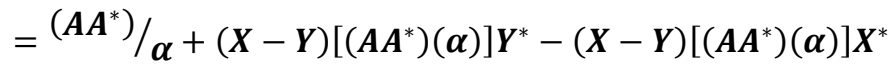

$$
\begin{aligned}
& =\left(\boldsymbol{A A ^ { * } )} / \boldsymbol{\alpha}+(\boldsymbol{X}-\boldsymbol{Y})\left[\left(\boldsymbol{A} \boldsymbol{A}^{*}\right)(\boldsymbol{\alpha})\right](\boldsymbol{X}-\boldsymbol{Y})^{*}\right. \\
& \geq\left(\boldsymbol{A A ^ { * } )} / \boldsymbol{\alpha}\right.
\end{aligned}
$$

is written. Similarly, the inequality

$$
(\boldsymbol{B} / \boldsymbol{\beta})(\boldsymbol{B} / \boldsymbol{\beta})^{*} \geq{ }^{\left(\boldsymbol{B} \boldsymbol{B}^{*}\right) / \boldsymbol{\beta}}
$$

can be shown. Then

$$
\begin{aligned}
& {[(\boldsymbol{A} / \boldsymbol{\alpha}) \otimes(B / \beta)][(A / \alpha) \otimes(B / \beta)]^{*}=[(A / \alpha) \otimes(B / \beta)]\left[(A / \alpha)^{*} \otimes(B / \beta)^{*}\right]} \\
& =\left[(\boldsymbol{A} / \boldsymbol{\alpha})(\boldsymbol{A} / \boldsymbol{\alpha})^{*}\right] \otimes\left[(B / \beta)(B / \beta)^{*}\right] \\
& \geq\left[\left(\boldsymbol{A A}^{*}\right) / \boldsymbol{\alpha}\right] \otimes\left[\left(\boldsymbol{B B}^{*}\right) / \boldsymbol{\beta}\right] \text { by Lemma } 2.5 \\
& \geq\left[\left(\boldsymbol{A} \boldsymbol{A}^{*}\right) \otimes\left(\boldsymbol{B} \boldsymbol{B}^{*}\right)\right] / \gamma \text {. }
\end{aligned}
$$

Hence Eq.(2), and therefore (4) follows. $\square$ 


\section{Conclusion}

We have obtained some new inequalities related to the block Kronecker products and Schur complements by using Albert's theorem. Using the above results similar studies can be improved for the singular values, eigenvalues, traces and determinants of Schur complements of block Kronecker products. Further, the upper or lower bounds of the spectral radius of matrices having an important place in matrix analysis, numerical analysis, and its applications can be investigated for the block Kronecker product of matrices.

\section{References}

[1] Brewer, J.W. 1978 Kronecker products and matrix calculus in system theory, IEEE Transactions on Circuits and Systems, Vol. Cas25, No.9.

[2] Horn, R.A., Mathias, R., and Nakamura, Y. 1991. Inequalities for unitarily invariant norms and bilinear matrix products, Linear and Multilinear Algebra, 30, 303314.

[3] Günther, M. \& Klotz, L. 2012. Schur's theorem for a block Hadamard product, Linear Algebra and its Applications, 437, 948-956.

[4] Horn, R. A. \& Johnson, C.R. 2013. Matrix Analysis, Cambridge University Press, New York.

[5] Liu, J. 1999. Some Löwner partial orders of Schur complements and Kronecker products of matrices, Linear Algebra and its Applications, 291, 143-149.
[6] Zhang, F. 2005. The Schur complement and its applications, Vol. 4, Springer Science \& Business Media.

[7] Albert, A. 1969. Conditions for positive and nonnegative definiteness in terms of pseudoinverses, SIAM J. Appl. Math. $17,434-440$. 